Please do not remove this page

RMIT

UNIVERSITY

\title{
An impulse detector for color image restoration
}

Phu, Meing; Tischer, Peter; Wu, Henry

https://researchrepository.rmit.edu.au/esploro/outputs/9921859922701341/filesAndLinks?institution=61RMIT_INST\&index=null

Phu, M., Tischer, P., \& Wu, H. (2006). An impulse detector for color image restoration. Proceedings of 2006 International Symposium on Intelligent Signal Processing and Communication Systems, 5-8.

https://doi.org/10.1109/ISPACS.2006.364824

Published Version: https://doi.org/10.1109/ISPACS.2006.364824

Repository homepage: https://researchrepository.rmit.edu.au

(C) 2006 IEEE

Downloaded On 2023/04/27 01:03:04 +1000

Please do not remove this page 


\title{
An Impulse Detector for Color Image Restoration
}

\author{
Mieng Quoc Phu*, Peter Eric Tischer ${ }^{*}$ and Hong Ren $\mathrm{Wu}^{\#}$ \\ "Clayton School of Information Technology \\ Monash University, Clayton Campus, Victoria, Australia, 3800. \\ \{mieng.quoc.phu | peter.tischer\} Amail.csse.monash.edu.au \\ "Software and Network Engineering, RMIT, City Campus, Victoria, Australia, 3001. \\ henry . wuermit .edu. au
}

\begin{abstract}
In this paper, a novel approach to impulsive noise detection is proposed for colour image restoration. First, a global Adaptive Region Growing scheme is used to separate uncorrupted clusters of pixels from corrupted clusters. Then, some Order Statistic Vector Filters are used to reconstruct the corrupted clusters. Various performance analyses show the detection scheme is more robust for a wide range of impulse noise than some of the state-of-the-art detectors. Thus, the proposed filter outperforms other filters in both objective and subjective image assessments.
\end{abstract}

\section{INTRODUCTION}

In the area of computer vision and image understanding applications, noise reduction plays a vital role in preprocessing the image.

Classic restoration filters designed specifically for greyscale images cannot be applied directly to color images because of the inter-channel correlation. Multivariate order statistics vector filters perform better and avoid drawbacks such as chromatic shifting and color bleeding [1]. Based on order statistic theory, a number of popular filters have been proposed in the last decade, among them are the popular vector median filter (VMF) [2], vector direction filter (GVDF) [3], directional-distance filter (DDF) [4], hybrid directional filter (HDF) [5] and the adaptive nearest neighbour filter (ANNF) [6]. These classic filters remove impulse noise adequately but they tend to destroy the image structure and blur thin lines and edges. The main reason for this is that they rely only on the order statistic theory. The center weighted vector filters (CWVF) [7, 8] have been proven to preserve detail better by giving extra weight to the central pixel. An advanced version of this filter is the selection center-weighted vector directional filter (SCWVDF) [9]. In order to remove impulse noise efficiently, a noise detection scheme should be carried out prior to the filtering process. The multiple window configuration (MWC) [10] and adaptive vector median filter (AVMF) [11] are examples of this type and proven to be very efficient. Other popular filters are the self adaptive algorithm (SAA) [12] and fast adaptive similarity filter (FAS) [13]. These filters can achieve high accuracy noise detection by utilizing adaptive online parameter tuning using a method known as Bisection. The peer group filter (PGF) [14] is a switch based filter which takes advantage of the local window statistics to segment pixels explicitly into groups with similar intensities. This filter is very robust for various impulse noise proportions.

A new detection switch-based filter is proposed in this paper. The filter uses a global region growing scheme in the detection process to help classify image pixels as 'clean' or 'noisy'. Then, a vector filter is used to reconstruct values for pixels corrupted by noise.

This paper is organized as follows. Section II describes the structure of the proposed filter and Section III is the parameter optimizations. In Section IV simulations and discussions are presented and finally in Section $\mathrm{V}$ the conclusions are given.

\section{THE PROPOSED FILTER STRUCTURE}

Fig. 1 shows the proposed filter structure. The proposed adaptive region growing vector filter (ARGVF), includes the region growing detection scheme (RGDS), the adaptive decision scheme (ADS) and the vector filter (VF) for reconstruction.

Let us define $I, O, S$ and $B$ as the input, output, cluster and binary images, respectively. This scheme creates a binary image, $B$, showing whether a pixel is corrupted or not. The replacement values of pixels marked as corrupted are computed by the VF.

For each pixel, the entry in the $S$ image shows to which cluster the pixel belongs and there are $K$ clusters in $I$. We further define $s_{k}$ be the number of pixels in cluster $C_{k}$ in image $S$, where k is the identification number of each cluster in $S$. For example, given an image $I$,

$$
\bigcup_{i=1}^{K} C_{i}=I, \quad C_{i} \bigcap_{i \neq j} C_{j}=0
$$

\section{A. Region Growing Detection Scheme}

The RGDS is the most important part of the proposed filter. Here, the input image is segmented into clusters of similar pixel intensity. The method used is generally known as region growing or technically we call it pixel-joining because only one pixel is added to a 'spatially coherent' cluster at a time. Spatially coherent means that only adjacent pixels are allowed to form clusters. For example, if we start with a cluster of one pixel, then the neighbouring pixels are examined in turn. If the neighbouring pixel's value is sufficiently close, i.e. 


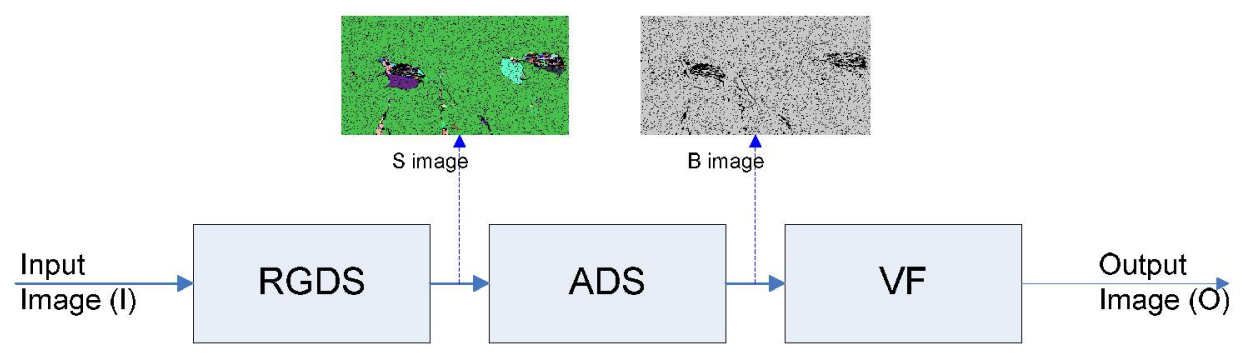

Fig. 1: The proposed ARGVF filter structure.

condition (2) is satisfied for two adjacent pixels where $\delta$ is a threshold and $\mathrm{N}$ is the number of neighbouring pixels, and it has not already been added to any cluster, it is added to the cluster and we then consider neighbours of that pixel. If we have run out of neighbours for the pixel which was added most recently, we return to the pixel added before that and continue to examine its neighbours. The process continues until all the pixels in a given image have been examined.

$$
\left\|x_{0}-x_{j}\right\|_{2} \leq \delta \quad j \in\{1, \ldots, N\}
$$

Notice, the size of cluster, $s_{k}$, is in a range $[1, \mathrm{H} \times W]$, where $H$ and $W$ are the height and width of the image, respectively. If every pixel in the image has at least one neighbour whose value is sufficiently close, then every pixel will be in the one cluster $\left(s_{1}=W \times H, k=1\right)$. However, the existence of impulse noise may mean that there are pixels in clusters with only a small number of elements. In general, uncorrupted pixels will be in clusters with large numbers of elements. These two remarks are the essence of our proposed detection technique. The RGDS algorithm can be implemented recursively.

\section{B. Adaptive Decision Scheme}

Once the RGDS process is finished, the ADS process determines which clusters are corrupted. Because impulse noise is randomly distributed over the whole image and with images containing a proportion of corrupted pixels less than $50 \%$, we have rarely observed cases where a cluster containing only corrupted pixels has more than 6 members. Our process assumes the pixels in clusters which have at least 7 members which are not corrupted. In addition, if a cluster contains only one pixel, we assume this pixel must be corrupt. Thus, if we assume the pixel of the binary image, $B$, take either the value 1 to represent noisy pixel or the value 0 to represent a clean pixel, then, the rules (3) and (4) always apply when filtering impulse noise with less than $50 \%$ corruption.

$$
\begin{aligned}
& \text { If } s_{k} \geq 7, \quad \forall x \in C_{k}, \quad x \text { is 'clean' } \\
& \text { If } s_{k}=1, \quad \forall x \in C_{k}, \quad x \text { is'noise' }
\end{aligned}
$$

For clusters with $2,3,4,5$ and 6 members we can classify them as being corrupt or clean depending on the proportion of pixels believed to be corrupted. Thus, we must adaptively adjust the condition depending on the estimated noise proportion $\phi_{e}$. From the data we collected on the randomness of impulse noise, we have formulated an adaptive approach to determine whether a cluster consists purely of noisy pixels. This switch based approach is shown in (5) and (6), where $s_{\max }$ is the maximum cluster size to be classified as 'noisy'. For example, if we estimated the noise proportion to be $10 \%$, i.e. $s_{\max }=3$, then all clusters of size less than 4 will be considered as noise. Using the decision rules in (5) and (6), all pixels can be classified as being either corrupted or clean and this can be stored in a binary image. To compute $\phi_{e}$, we used equation (7). We assume that all pixels in a cluster with only 1 or 2 members are corrupted and we use the total number of pixels in these clusters to determine an estimated proportion.

$\phi_{e}$ is a good estimate of the actual input noise, but as noise increases this estimate worsens because the number of noise clusters which have more than 2 members increase proportionally.

$$
\begin{gathered}
s_{\max }= \begin{cases}1 & \phi_{e}<0.9 \% \\
2 & 0.9 \leq \phi_{e}<9.6 \% \\
3 & 9.6 \leq \phi_{e}<23 \% \\
4 & 23 \leq \phi_{e}<31 \% \\
5 & 31 \leq \phi_{e}<39 \% \\
6 & \phi_{e} \geq 39 \%\end{cases} \\
\text { If } s_{k} \leq s_{\max }, \quad \forall x \in C_{k}, \quad x \text { is }^{\prime} \text { noise' }
\end{gathered}
$$

\section{Vector Filters}

The reconstruction filter is only used if the centre pixel is corrupted. For reconstruction, any vector filter can be used. We chose VMF[2], VDF[3] and ANNF[6]. Let ARGVMF, ARGVDF and ARGAANF denote the proposed filters which use VMF, VDF and ANNF filters for reconstruction, respectively.

\section{OPTIMIZATION OF DELTA}

From the RGDS section, the threshold $\delta$ from equation (5) is very important. Unfortunately there is no fixed value for it because every image is different in nature and how we perceive the similarity in colors also varies from one person to another. The effects of $\delta$ on the cluster image $S$ and on $B$ are shown in Fig. 2. For small values of $\delta$, more clusters are formed and the number of small clusters increase significantly. 
This occurs mainly at the edges due to many variations of pixel intensity. Thus, the RDGS often misclassifies clean pixels as noisy pixels. In contrast, for large values of $\delta$, fewer clusters with more pixels are formed. This in effect will misclassify noisy pixels as being clean pixels.

In order to find a compromise value for $\delta$, we used two noise detection error measurements. Let us define FP as the number of clean pixels falsely classified as noisy and FN as the number of noise pixels falsely classified as clean. Fig. 3 shows for a range of $\delta$ values, a good compromise value is where the FN and FP intersect (Note - the vertical scale is logarithmic). For the Parrot image with 10\% of impulse random noise, the compromise value is 50. Also shown in Fig. 3 , if our aim is to alleviate impulse random noise (i.e. minimize FN), then we should choose a threshold value lower than the compromise value. However, if our aim is to preserve image structures (i.e. minimize FP) then we should make the threshold higher than the compromised value. Our extensive experiments show that if $\delta$ is in the range of 25 to 60 , the proposed filters are very efficient for natural images. In this paper, we used $\delta=40$ as the compromise value for all natural images and a wide range of impulse noise levels.
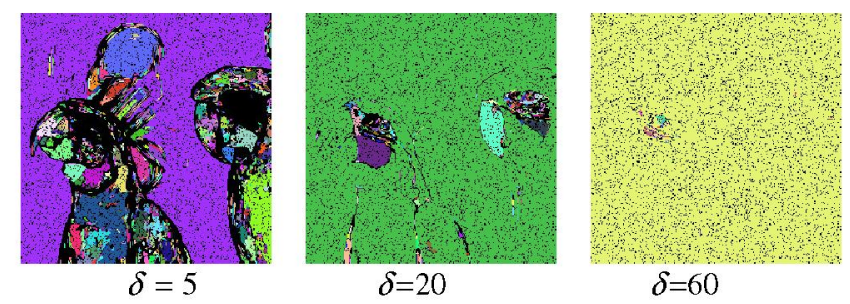

Fig. 2: The effects of varying $\delta$ on the $\mathrm{S}$ image.

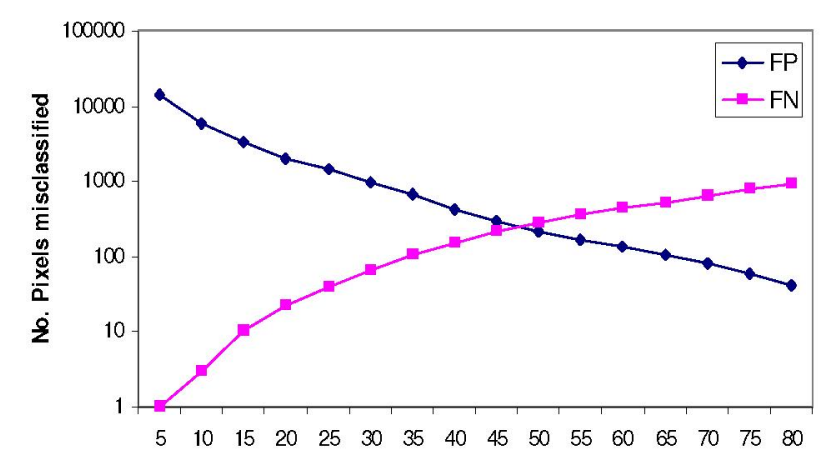

Fig. 3: The value of $\delta$ where the FP (False Positive) and FN (False Negative) intersect is the compromise value for the Parrots image with $10 \%$ random impulse noise.

\section{COMPARATIVE RESUlts}

The impulse noise corruption for color images in this paper is modeled using the same method as $[9,11,12]$. Several objective criteria are used to test the efficiency of the proposed filter, which includes mean square error (MSE), mean absolute error (MAE) and normalized color difference (NCD). NCD is scaled by $10 \mathrm{e}^{-2}$. These are the most frequently used measurements in the literature and are defined by Lukac[9].

\section{A. Subjective and Objective Assessments}

In this section, the evaluations of the proposed filters on color images are presented. The proposed filters ARGVMF, ARGVDF and ARGANNF are compared with some classic filters as well as the state-of-the-art filters such as AVMF, SAA and the PGF filters. All parameters are as recommended by the referenced authors. Additionally, we used a fixed window size of $3 \times 3$ for all filters. The Lena and Peppers images are $512 \times 512$ in dimension, except for the Parrots image which is $256 \times 256$. They are corrupted by random impulse noise at $10 \%, 25 \%$ and $40 \%$.

Table I summarizes the objective performance measures of the proposed filters compared with state-of-the-art filters on the three images. It can be seen that all switch-based filters (AVMF, SAA and PGF) outperform the classics filters, such as VMF and ANNF filters. The results show that the proposed filters ARGVMF, ARGANF and ARGVDF have achieved better performance than most filters. The ARGVMF which uses the VMF for reconstruction improved by a significant amount compared to the classic VMF. For the Lena image at $10 \%$ corruption, the ARGVMF is 5.4 times better than the VMF in NCD measurement. In addition, the ARGVMF is much faster than VMF in speed because it uses the VMF only if the pixel is classified as noise. The ARGVMF also outperforms all other filters in various degrees. As noise level increases, advanced filters such as AVMF, SCWVDF and SAA perform significantly worse but the ARGVMF gives acceptable results. Table I also shows that for a single $\delta=40$ threshold, the proposed filter is efficient for various proportions of noise and on most standard test images. We found that for delta between 25 and 60 , the proposed ARGVMF performed well. Fig. 4 demonstrates the reconstructed images of the Parrots at $25 \%$ corruption. The noise-free ARGVMF image shows that the proposed filter preserves the image structure well. The AVMF and SAA filters preserved the image structures well but have a fair amount of residual noise in the reconstructed image. As noise level increases, the number of residual noise pixels also increases

\section{CONCLUSIONS}

A novel and fast global adaptive region growing vector filter is proposed in this paper for color image restoration. The ARG approach leads to methods which are quick since the reconstruction filter is only applied to noisy pixels and the noise identification procedure is also quick. The proposed noise detector performs well for various proportions of random noise. The results show the detector is very robust and achieved high accuracy in removing noise while preserving image structure. Subjective and objective assessments confirmed that on standard test images, the proposed filter is superior to the state-of-the-art filters. 


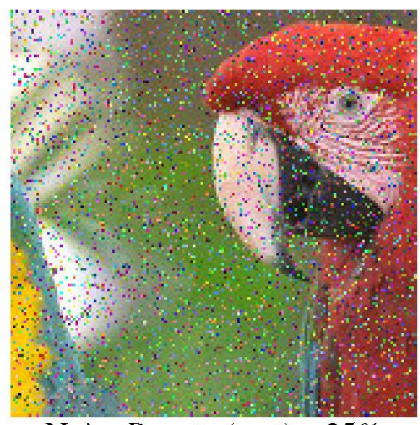

Noisy Parrots (part) $-25 \%$

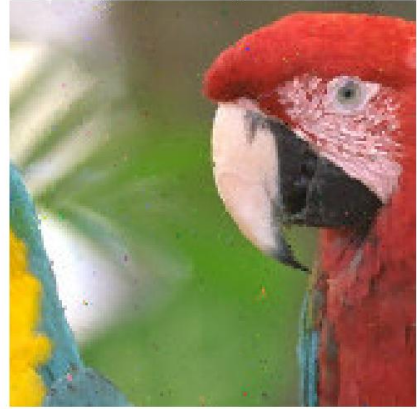

PGF $(\mathrm{NCD}=1.499)$

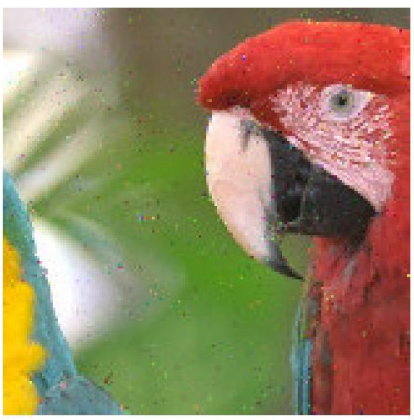

SAA $(\mathrm{NCD}=2.198)$

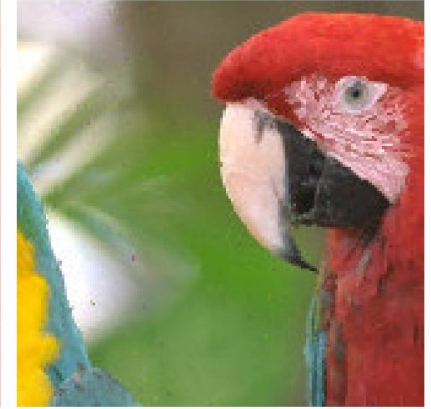

$\operatorname{ARGVMF}(\mathrm{NCD}=1.279)$

Fig. 4: Reconstruction of the proposed ARGVMF filter compared with the SAA and PGF filters. The Parrots was corrupted by $25 \%$ of random impulse noise.

\section{REFERENCES}

[1] K. N. Plataniotis, A. N. Venetsanopoulos (Eds.), Color Image Processing and Applications, Springer, Berlin, 2000

[2] J. Astola, P. Haavisto, Y. Neuov, Vector median filter, Proc. IEEE 78(4)(1990) 678-689.

[3] P. E. Trahanias, A. N Venetsanopoulos, Vector directional filters: a new class of multichannel image processing filter, IEEE Trans. Image Processing 2(4) (1993) 528-534.

[4] D. G. Karakos, P. E. Trahania, Combining vector median and vector directional filters: the directional-distance filter, Proc. IEEE Int. Conf. Image Processing (ICIP'95), Vol. 1, Washington D. C, (1995) 171-174.

[5] M. Gabbouj, F. A. Cheikh, Vector median-vector directional hybrid filter for color image restoration, Proc. EUSIPCO, Vol. 2, Trieste, Italy, (1996) 879-881.

[6] K. N. Plataniotis, et al., An adaptive nearest neighbor multichannel filter, Trans. IEEE, Circuit Sys., Video Tech., 6(6), (Dec. 1996) 699-703.

[7] T. Viero, K. Oistamo, Y. Neuvo, Three-dimensional median-related filters for color image sequence filtering, IEEE Trans. Circuits Syst. 4(2) (1994) 129-142.

[8] R. Lukac, K. N. Plataniotis, B. Smolka, A. N. Venetsanopoulos, Generalized selection weighted vector filter, EURASIP Journal on Applied Signal Processing 2004 (12) (2004) 1870-1885.

[9] R. Lukac, Adaptive color image filtering based on center-weighted vector directional filters, Multichannel Sys. Signal Process. 15(2) (2004) 169-196.

[10] E. S. Hore, B. Qiu, H. R. Wu, Prediction based image restoration using a multiple window configuration, Optical Engineering, Vol. 41, (Aug. 2002) 1-11.

[11] R. Lukac, Adaptive vector median filtering, Patt. Recogn. Lett. 24 (2003) 1889-1899.

[12] B. Smolka, et al., Self-adaptive algorithm for impulsive noise reduction in color images, Patt. Recogn. 35(8) (2002) 1771-1784.

[13] B. Smolka, et al., Fast adaptive similarity based impulse noise reduction filter, Real-Time Imaging, Special Issue on Spectral Imaging 9(4) (2003) 261-276.

[14] C. Kenney, et al., Peer group image enhancement, IEEE Trans. Image Processing, 6(7), (Feb 2001) 326-334.

TABLE I

THE PERFORMANCE OF THE STATE-OF-THE-ART FILTERS COMPARED WITH THE PROPOSED ARGVF, FOR 10\%, 25\% AND 40\% OF RANDOM IMPULSE NOISE.

\begin{tabular}{|c|c|c|c|c|c|c|c|c|c|c|}
\hline \multirow{2}{*}{ Images } & \multirow{2}{*}{ Filters } & \multicolumn{3}{|c|}{ Input Proportion: $10 \%$} & \multicolumn{3}{|c|}{ Input Proportion: $25 \%$} & \multicolumn{3}{|c|}{ Input Proportion: $40 \%$} \\
\hline & & MSE & MAE & NCD & MSE & MAE & NCD & MSE & MAE & NCD \\
\hline \multirow{5}{*}{$\begin{array}{c}\text { Lena } \\
(512 \times 512)\end{array}$} & VMF & 34.8 & 3.446 & 3.998 & 47.8 & 3.950 & 4.494 & 78.3 & 4.684 & 5.301 \\
\hline & ANNF & 33.6 & 3.820 & 4.430 & 59.7 & 5.151 & 6.173 & 112.3 & 7.081 & 8.687 \\
\hline & SAA & 17.7 & 0.660 & 0.937 & 65.2 & 1.884 & 2.714 & 118.7 & 3.104 & 4.382 \\
\hline & PGF & 11.4 & 0.619 & 0.782 & 34.2 & 1.518 & 1.938 & 75.6 & 2.653 & 3.384 \\
\hline & ARGANF & 10.3 & 0.596 & 0.778 & 32.1 & 1.728 & 2.268 & 71.6 & 3.333 & 4.348 \\
\hline \multirow{5}{*}{$\begin{array}{c}\text { Peppers } \\
(512 \times 512)\end{array}$} & VMF & 46.5 & 4.114 & 5.179 & 64.0 & 4.618 & 5.779 & 113.8 & 5.522 & 6.998 \\
\hline & ANNF & 43.8 & 4.440 & 5.690 & 75.9 & 5.817 & 7.878 & 151.0 & 8.061 & 11.266 \\
\hline & AVMF & 21.3 & 0.768 & 1.040 & 50.4 & 1.839 & 2.506 & 109.5 & 3.244 & 4.446 \\
\hline & SAA & 21.5 & 0.738 & 1.038 & 72.9 & 2.023 & 2.870 & 147.1 & 3.452 & 4.853 \\
\hline & PGF & 15.9 & 0.698 & 0.909 & 42.4 & 1.686 & 2.250 & 102.3 & 3.035 & 4.091 \\
\hline \multirow{7}{*}{$\begin{array}{c}\text { Parrots } \\
(256 \times 256)\end{array}$} & $\mathrm{ANNF}$ & 58.4 & 3.235 & 2.271 & 90.9 & 4.874 & 4.472 & 152.2 & 7.104 & 7.375 \\
\hline & AVMF & 44.7 & 1.029 & 0.814 & 75.2 & 1.945 & 1.854 & 126.0 & 3.099 & 3.199 \\
\hline & SAA & 37.0 & 0.757 & 0.751 & 86.9 & 1.872 & 2.198 & 146.5 & 3.004 & 3.550 \\
\hline & PGF & 23.6 & 0.781 & 0.634 & 58.4 & 1.658 & 1.499 & 109.2 & 2.672 & 2.557 \\
\hline & ARGANF & 21.0 & 0.679 & 0.598 & 56.0 & 1.966 & 1.990 & 105.5 & 3.690 & 3.979 \\
\hline & ARGVDF & 30.5 & 0.737 & 0.515 & 74.9 & 1.781 & 1.320 & 136.8 & 2.958 & 2.300 \\
\hline & ARGVMF & 20.9 & 0.614 & 0.492 & 52.1 & 1.532 & 1.279 & 93.6 & 2.569 & 2.255 \\
\hline
\end{tabular}

